\title{
Del pan y del vino en un texto médico del siglo XIV
}

\author{
JOSÉ MONDÉJAR \\ Dpto. de Historia de la Lengua Española. \\ Universidad de Granada
}

\section{RESUMEN}

Este artículo trata de dar a conocer lo que del pan y del vino medicinal nos dice Juan de Aviñón en su tratado de medicina galénica, Sevillana medicina (Sevilla, 1545). Sus opiniones personales, en medio de las heredadas en los tratados anteriores, interesantes y curiosas siempre, se refieren, generalmente, a lo que se produce, elabora y se consume en Sevilla (alguna referencia esporádica a lo que se hace en su Francia natal, no deja de tener interés). Tanto el pan como el vino medicina son analizados en su naturaleza y en sus efectos en el proceso de la digestión, así como desde el punto de vista de la oportunidad de su consumo de acuerdo con el trabajo que se tenga, sobre todo en el caso del pan.

Palabras clave: Medicina, Onomasiología, Lexicología, Pan, Vino.

\section{SUMMARY}

The aim of this article is to communicate what is said in Juan de Aviñón's Galenic medicine treatise Sevillana Medicina (Seville, 1545) about bread and medicinal wine. Within the frame of traditional views taken from previous treatises, his own opinions are interesting and remarkable and they usually refer to what is produced and consumed in the city of Seville (Some occasional references to what is made in his birthplace, France, do not lack interest). Bread and medicinal wine are examined with regard to their nature, as well as to their effect on the process of digestion. At the same time, they are considered from the point of view of the appropriateness of their consumption according to the consumer's employment, especially with reference to bread.

Key words: Medicine, Onomasiology, Lexicology, Bread, Wine.

\section{INTRODUCCIÓN}

Gracias a la diligencia y actividad humanística del médico sevillano Nicolás Monardes (h. 1507-h. 1588) (Lasso de la Vega 1988; Rodríguez

RDTP, LVII, 2 (2002): 167-188 
Marín 1988), conocemos la existencia de una obra de excepcional importancia en la historia de la galénica medieval española: la Sevillana Medicina (1384). Se publicó a instancias de este benemérito sevillano, por el Concejo de la ciudad de Sevilla, en 1545.

El texto de esta obra, singular en bastantes aspectos, nos ha llegado en tal estado de deturpación que me parece imposible creer que haya sido entendida en su totalidad por cuantos han opinado sobre ella. Sólo un trabajo filológico paciente de restauración de su traslado al castellano, ha hecho posible, en buena medida, comprender lo que el autor quiso decir en un texto latino, del que no se ha conservado ni siquiera una copia, como testimonio de sus conocimientos médicos.

La impresión sevillana pretende ofrecer el texto castellano de la traducción del manuscrito latino, muy probablemente hecha en el mismo siglo XIV, lo que, en general, en cierta medida consigue, habida cuenta del mal estado en que llegó a manos de Monardes, a lo que hay que añadir las prevaricaciones del impresor (Aviñón 2000).

Lo que conocemos de su autor, el converso conocido con el nombre cristiano de Juan de Aviñón, y de nombre judío Mo e ben Samuel, natural de Roquemaure (Dept. de Gard), en el Languedoc, directa o indirectamente nos lo proporciona esta obra. Debió de nacer entre 1323 y 1328 y, como quiera que alcanzó el reinado de Juan I (1379-1390), moriría, probablemente, con la edad de 62 o 67 años, en Sevilla, adonde vino a parar, como médico del arzobispo Gómez Barroso, en 1353.

El contenido de la Sevillana Medicina (en adelante, SMed.) tiene poco de original, salvo los seis capítulos dedicados al estudio del aire de Sevilla (II-VII), los párrafos dedicados a la "diamagna", fármaco por él confeccionado en 1360, de propiedades curativas asombrosas, al decir de Aviñón, y alguna receta culinaria; no obstante, la falta de originalidad es achaque propio de esta clase de obras medievales, lo que no viene en demérito de ninguna de ellas y, mucho menos, de la que hoy nos ocupa, por la abundancia de noticias que de Sevilla y otras partes de España y Francia, respecto de costumbres, hábitos culinarios y productos tiene.

Entre ellas, se encuentran algunas concernientes a las clases de trigo, de harina, de pan, de uvas y de vino, de Sevilla; incluso de vinos medicinales, que son los que estudiaremos.

\section{DE LAS CLASES DE TRIGO}

En Sevilla, según Aviñón, hay varias clases de trigo, respecto de las cuales hace veintidós observaciones: 
El catamie $<$ n $>$ to $p<$ ri $>$ mero $e<n>$ la color, ca trigo ay amarillo como la cera, de fuera, y bla $<\mathrm{n}>\mathrm{co}$, de de $<\mathrm{n}>$ tro, y lezino y claro y ligero de $\mathrm{q}<\mathrm{ue}>$ brar y con poco afrecho y mucha farina; y este tal es de gran nudrimie $<n>t o$, pero es pesado de moler y $\mathrm{d}<\mathrm{e}>$ ello ay que es el $\mathrm{co}<\mathrm{n}>$ trario; $\mathrm{y}$, aqui, en Seuilla ay de muchas maneras de trigo: ay gazul, ay semental, ay bermejuelo, [f. 27r] ay aluarigo, ay trechel, ay delgado; las tres $\mathrm{p}<\mathrm{ri}>$ meras son las mejores y el gazul es el mejor $\mathrm{d}<\mathrm{e}>\operatorname{todos}^{1}$.

De todas estas clases de trigo que Aviñón menciona, las de "semental" y "delgado" son tan generales, que en la práctica no significan nada, porque, en principio, todos los trigos pueden sembrarse y porque muchos de ellos son de grano delgado; el "bermejuelo" es de difícil identificación, a no ser que se trate del "rubión", que Columela llama [triticum], quod robus dicitur ${ }^{2}$, como les ocurre a todos los caracterizados por el color, así "amarillento", "dorado", "azulenco", y algunos más, pero no a "blanco" o "albarigo", porque mientras que los primeros se refieren al color del hollejo, que molido el grano se conoce con los nombres de "afrecho" o "Salvado", los dos segundos se dan al de la "harina" por su extremada blancura; así es que la nómina queda reducida a "gazul", "albarigo" y "trechel". Además, la mayor parte de las denominaciones que s.v. trigo recoge el DRAE son de uso regional e, incluso, local, y no digamos las que da el DPA (Sánchez-Monge 1981: s.v. trigo: 349-350), entre las que se encuentran algunas tan "castizas" como einkorn, maka y poulard; por el contrario, apenas algunas de las seleccionadas pertenecen al español común, así "candeal" o "albarigo", "desraspado" o "chamorro", "trechel" o "tremesino", "fanfarrón", etc.

El trigo que Aviñón califica de "lezino" tiene, entre otras cualidades, todas positivas, una negativa: que es de difícil digestión, frente a otras especies, lo que no deja de ser un asunto secundario para un filólogo. Lo importante es el calificativo de "lezino", cuya existencia conocemos por la SMed., que da a una determinada especie de trigo, cuyas características describe; el testimonio de "lezino" recogido en el DETEMA (Herrera 1996) s.v. lezne, 2., es este de la SMed., por no conocerse otro más antiguo. Dicho esto, cabe preguntarse: ¿qué especie de trigo es ésta tan alimenticia, tan generosa de harina blanca y tan escasa de salvado y quebradizo:

ca trigo ay amarillo como la cera, de fuera, y bla $<n>c o$, de de $<\mathrm{n}>$ tro, y lezino y claro y ligero de $q<u e>b r a r$ y con poco afrecho y mucha farina?

\footnotetext{
${ }^{1}$ La cursiva de los textos es mía.

2 Texto de Columela: "Tritici genera complura cognovimus, verum ex iis maxime serendum est, quod robus dicitur, quoniam et pondere et nitore praestat" (Columella 1977: I, II, vi, 136).
} 
Se me ocurre que, según la descripción dada, no puede ser otro que el "candial" de Berceo: la Virgen se lleva el alma del pobre al Paraíso: "do se ceban los angeles del buen candial trigo" (137c), cuyo sinónimo específico es el "aluarigo" del texto. El trigo de este nombre lo cacterizó por vez primera y de manera relativamente cumplida Covarrubias en su Tesoro (1943):

Una especie de trigo que haze el pan muy blanco y regalado, latine siligo ${ }^{3}$ candeal; y el mesmo pan se haze de la flor de la harina, y le dan el mesmo nombre y le llaman pan candeal. Dixose assi a caln]dore, panis siligineus.

Sinónimos de "candeal" son, pues, "blanco" y "albarigo". En cualquier caso, se trate o no del trigo "candeal", en el texto de Aviñón, el "albarigo" que se cosechaba en las tierras de Sevilla, aunque no lo supiera, está en lugar del "candeal" descrito; por otra parte, el galeno sevillano, nacido en el Languedoc, al señalar las peculiaridades del excelente trigo parece incurrir en redundancia, al decir que es "lezino" y "ligero de quebrar"; pero no, aquí "lezino" vale por 'tierno', porque hay trigos de grano 'duro'.

El lat. LENIS 'dulce' al tacto, es decir, 'suave', y en sucesivos desplazamientos semánticos romances a campos léxicos próximos, 'ligero' 'tierno' 'quebradizo'. El DCECH s.v. deleznarse, del más antiguo deslenarse, da "lezne", del ant. "lene", por influjo de la forma del infinitivo. Ahora bien, la variante léxica primera y directamente sacada por el hablante es la de "lezna", que no recoge el DCECH, como tampoco "lezino", adj. derivado de "lezne". El DCECH también desconoce la existencia de "gazul", y de "bermejuelo".

El adj. "gazul" (1384) sólo se encuentra en Andalucía, pues así lo confirma, además del texto galénico sevillano, frente a su ausencia en otros que tratan el mismo asunto, la documentación lexicográfica en la entrada garzul, cuyo más antiguo registro aparece en el DRAE 'adj. $p$. And. Se aplica a cierta especie de trigo. Tritici genus ( $\left.{ }^{4} 1803\right)$. También se alfabetiza "garzul" en el Vocabulario de Alcalá Venceslada (1980), único que lo hace, entre los muchos de naturaleza regional o local que he consultado. Lo registran, por el contrario, los diccionarios generales de Núñez Taboada (1825): ‘adj. And. Dicese de cierta especie de trigo'; Salvá (1846),

3 Dice Francisco Hernández (1976: nota 15, cap. vii, libro XviII, respecto de candeal: "Aunque siligo se toma algunas vezes por la flor, o parte regalada de la harina, como polen es lo que se pega a las paredes, y simila o similago la sémola que llamamos acemite, y pan autopyro lo que se haze de todo el grano, quiere en otra significación dezir un género de panes que en Hespaña parten en cuatro o más diferencias, llamando uno tremesino, otro candeal universalmente y por eso lo interpreté desa manera". 
Domínguez (1853), entre otros muchos; pero el primero que no le da entrada, y que para documentarlo remite a trigo, es Zerolo (1895); esta especie de trigo era o, todavía, es conocida en distintas regiones de España con el adj. de "álaga" (<lat. ALICA 'escanda'): 'especie de trigo. Latine alica, ab alendo, quod vehementer nutriat' (Tes., s.v. 1611); y quien por vez primera dió "álaga" como sinónimo de trigo "garzul" fue Pagés s.v. trigo, en el último vol. de su diccionario (1931), pero registra la entrada "álaga" en el primer vol. (1902); esta identificación se repite hasta la penúltima edición del DRAE (21992). La "escanda" es una especie de trigo propia de tierras frías y pobres, de caña dura y corta, cuya harina es difícil de separar del afrecho ( $\left.{ }^{22} \mathrm{DRAE}\right)$. La primera doc. académica de "álaga" es de 1803:

Especie de trigo, que produce un grano largo, que amarillea: el pan que se hace de él tira al mismo color, y es dulce y de poca corteza' (DRAE s.v.);

prudentemente, en la última edición, sólo se dice:

Especie de trigo, muy parecido al fanfarrón, que produce un grano largo y amarillento ( ${ }^{22}$ DRAE S.v., 2001).

En la Sevillana Medicina, la forma original del adj. es "gazul", que yo rectifiqué en "garzul", en la edición que de la misma hice (Aviñón 2000), siguiendo la ortografía dada en su primer registro lexicográfico (DRAE, 1803). Ahora, esta rectificación, por parte de la Academia, convierte "garzul" en un fantasma, porque además de ser "gazul" la única documentada, y siendo exclusiva del sur de España ¿no podría tratarse de una especie de trigo "Candeal" famosa por su calidad, cultivada en Alcalá de los Gazules (Cádiz)? De donde "candeal", "blanco", "albarigo" y "gazul", serían cuatro especificaciones adjetivas de la misma clase de trigo, aunque, las tres primeras, por distinta motivación que la última.

De todos los trigos que se sembraban en Sevilla, en el s. XIv, el "gazul" "es el mejor de todos", a juicio de Aviñón.

En cuanto a que el trigo "álaga" sea el mismo que el "garzul" lexicográfico, me parece un error: "garzul. And. trigo álaga" ( ${ }^{21} \mathrm{DRAE}, 1992$, s.v. trigo; en la ${ }^{22} \mathrm{DRAE}$, el pretendido andalucismo "garzul", ha desaparecido, justificadamente, porque jamás se ha tenido noticia de su documentación). Según este mismo diccionario, el grano del trigo "álaga" (Rosal 1601) (DAut. 1726), es muy parecido al del "fanfarrón" (DRAE, s.v., 1869):

Es procedente de Berbería, duro, crece muy alto, de espigas arqueadas largas: da mucho salvado y rinde por lo mismo menos pan, aunque de buena calidad: abunda mucho en Andalucía; 
luego no parece que sea un buen trigo, ya que se trata de un cereal que, especialmente, se estima por la cantidad y blancura de su harina y escasez de afrecho, así el "candeal", que es el trigo que, comunmente, se utiliza para hacer el pan. ¿Cómo podría, entonces, decir Aviñón que el mejor grano de trigo, si no de Andalucía, de Sevilla, es el "gazul", si se pareciera al "álaga" y al "fanfarrón", como quiso el DRAE, hasta 1992, y como quiso la lexicografía decimonónica de autor?

Respecto de "trechel" (1384), denominación cuyo origen es mozárabe y cuya base sería el ordinal latino TERTIARIUS> *tercher, según el DCECH s.v.

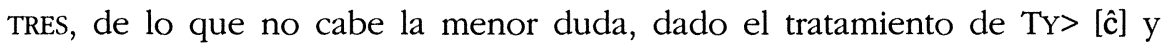
del suf. -ARIUS>-ayr> -eyr $>-e r /-e l$, es una variedad de trigo bien conocida en los textos de distinta procedencia, no siempre igualmente estimada, y cuya maduración dura 'tres meses'. Si para el converso Aviñón esta especie no está entre las tres mejores: "gazul", "Semental" y "bermejuelo", de las seis que menciona, sí es la mejor para Alonso de Herrera (1981: 60b):

Ay un trigo trechel que llaman rubion: esto es lo mejor de todo, assi en peso como en provision.

Para el DCECH, la primera datación literaria se encuentra en esta obra de Agricultura (1513), cuando aparece en la SMed. (1384); por lo que respecta a la lexicográfica, se halla en Del Rosal (1601).

Todavía queda otro punto y no de escasa importancia por lo que a la sinonimia de "trechel" se refiere. El DRAE s.v. trigo da como sinónimos "tremés" o "tremesino", sinónimos que responden al significado de la base, y que por ello, naturalmente, el DCECH no discute; este tipo de trigo, hoy, en general, se conoce con el nombre de "temprano", pues se siembra en primavera y se recoge en el verano del mismo año, antes o después, dependiendo, claro está, de la latitud del punto de referencia. Ahora bien, "trechel" es una denominación genérica que afecta a todas las variedades de trigo temprano, una de las cuales es el "rubión".

Para Alonso de Herrera (1981: 60b), que no supo de etimologías, el "tremesino", sin embargo, no tiene nada que ver con otras especies de trigo, por ejemplo el "trechel":

Ay un trigo trechel que llaman rubion ${ }^{4}$ : esto es lo mejor de todo, assi en peso como en provision. Ay otro que llaman arisprieto, y esto es muy vecino al trechel. Ay otro trigo blanco, o candeal. Ay de[s] raspado y tremesino.

${ }^{4}$ El traductor de Lucio Junio Moderato Columela (1988: II, cap. 6, nota 24, p. 48) dice que "Es una variedad de trigo fanfarrón, de grano dorado", en contra de lo que opina Alonso de Herrera. Columela se limita a decir: [triticum], quod robus dicitur. Siguiendo el parecer de Herrera, creo que se trata de una variedad de trigo atrecheln y no de "fanfarrón". 
Según la RAE, ${ }^{22} \mathrm{DRAE}$, "rubión" también es 'variedad de trigo fanfarrón'.

Qué duda cabe de que "trechel", "tremés", "tremesino" y "marzal" significan lo mismo, por lo que discutir si "tremesino" es especie distinta de la de "trechel", es cosa vana, porque sea cual sea la especie que se siembre (generalmente en primavera y se recoge en verano), si se cosecha a los tres meses es "trechel"; pero, en la Antigüedad, Plinio disintió de Columela por no creer el gaditano que fuera una variedad de trigo distinta de otras aquel que madurara y se pudiera cosechar tres meses después de sembrarse. Cuando Herrera reproduce la opinión de ambos, en lo que a Columela se refiere, dice que incurre en contradicción, porque si en una página afirma que para el hispanorromano uel tremesino es manera de simiente apartada de todos los otros trigos" (61a), o lo que es lo mismo, distinta de las demás, en la siguiente afirma que "Columela dice (como arriba alegué) que la simiente tremesina no es simiente propia, ni [se] diferencia de otros trigos" (62a). Lo que Columela estima, según el texto latino y la traducción que utilizo, es todo lo contrario. En la breve clasificación que, de acuerdo con su calidad, hace, el primero es el "rubión", el segundo el "candeal" y el tercero el "tremesino":

En tercer lugar, está el tremesino, cuyo uso es muy socorrido para los agricultores, pues, cuando por las aguas o por otra razón no se ha sembrado a tiempo, recurren a él. Es, en realidad, una variedad del candeal (Columela 1988: cap. 6, $34 a)^{5}$.

Y tres capítulos más adelante, confirma lo que acabo de transcribir:

Pues no hay, como muchos han creído, semilla alguna tremesina por naturaleza, puesto que esa misma semilla, sembrada en otoño, responde mejor (Ibid. cap. 9, $35 b)^{6}$.

Así pues, no sólo no hay contradicción en lo que Columela dice, sino todo lo contrario, máxima coherencia.

Añade Herrera, siguiendo a Plinio, que esta clase de simiente es "propia de tierras frías y nevosas" (62a).

En la traducción que Francisco Hernández hizo de la Naturalis Historia (1576) de Plinio se dice lo que sigue:

5 Texto de Columela: "Id porro genus est siliginis" (Columella 1977: I, II, vi, 2, 136-137). Vid. nota 3, en la que Francisco Hernández sostiene opinión distinta, respecto de la diferencia entre "Candeal" y "tremesino", en el español de su tiempo.

${ }^{6}$ Texto de Columela: "Neque enim est ullum, sicut multum crederunt, natura trimestre semen, quippe idem iactum autumno melius respondet (Columella 1977: I, II, $i x, 8,146-148)$. 
También hay diferencia en la caña, porque la más gruesa es de mejor género. Vístese el trigo de Thracia de muchas telas, buscando ansí contra los fríos de aquella región. Por la misma causa hallo los tremesinos, ocupando las tierras la nieve, el cual se siega ${ }^{7}$ solos tres meses después de sembrado, y este pan es común a todos los Alpes y provincias frías; no hay linage de trigo de mejor vista que aqueste $^{8}$.

\section{Continúa Hernández:}

Tan grande es el engaño que Columella recibe, el cual no creyó haver género de trigo aun de tres meses, como sea antiquísimo y lo llamen los griegos trimeno $(315 a)^{9}$.

Lo que Columela negó es que el "tremesino" fuera una especie; lo que afirmó es que era "una variedad del candeab.

\section{DEL PAN DE TRIGO}

\section{La harina}

Aviñón hace veintidós observaciones respecto del pan de trigo; la número once se refiere a las tres clases de harina, conocidas en Sevilla: la "Seca", el "almodón" (no hasta DAut., 1726) y la "adárgama" (no hasta DAut., 1726):

El.$x j$. catamie $<n>$ to es segu $<$ n $>$ la manera $d<e>$ la farina $q<u>e$ ay aq $<\mathrm{ui}>$, $d<e>l l a$ [ay] de tres maneras: farina seca y almodon y adargama; y la farina seca $\mathrm{q}<\mathrm{u}>\mathrm{e}$ no $<\mathrm{n}>$ fue rociado el trigo, es buelta $c o<n>$ afrecho $y$, por ende, es mas liuiana de dece $<\mathrm{n}>$ dir $\mathrm{d}<\mathrm{e}>\mathrm{l}$ estomago y de cozer mas ayna, mas non es de tal gouierno ni ta $<\mathrm{n}>$ buena como las ${ }^{10}$ otras ${ }^{11}$; [el trigo del] ${ }^{12}$ almodon remojanlo en agua y muelenlo despues gruessame $<n>t e$ y despues tiran aq $<\mathrm{u}>\mathrm{el}$ afrecho gruesso y lo $\mathrm{q}<\mathrm{u}>\mathrm{e}$ finca es muy bueno y faze muy buen pan y sabroso, e porq $<\mathrm{u}>\mathrm{e}$ ay algun afrecho que es liuiano de moler y de comunal nudrimie<n $>$ to; el adargama es,

\footnotetext{
7 Por errata, en el texto, siembra.

${ }^{8}$ Texto de Plinio (1971: XVIII, 234): «[...]. eadem causa et trimestre invenit detinentibus terras nivibus quod tertio fere a satu mense cum et reliquo orbe metitur. $[\ldots]$.

9 in tantum fallitur Columella qui ne trimestri quidem proprium genus existimaverit esse, cum sit antiquissimum" (Plinio 1971).

${ }^{10}$ las: los

11 otras: otros

${ }^{12}$ Lo añadido va entre corchetes y letra redonda; lo que se suprime, entre corchetes y cursiva.
} 
remojado ${ }^{13}$ y molido ${ }^{14}$ gruessamente cada grano en tres partes o $\mathrm{q}<\mathrm{ua}>\mathrm{tro}, \mathrm{y}$ despues tiran dende aq $<\mathrm{u}>\mathrm{el}$ afrecho largo y gruesso, y finca $<\mathrm{n}>15$ aq $<\mathrm{u}>\mathrm{ellos}^{16}$ gra $<\mathrm{n}>\mathrm{os}^{17}$ limpios ${ }^{18}$ [de] gra $<\mathrm{n}>$ ças [f. 28r] y descortezados ${ }^{19} \mathrm{~d}<\mathrm{e}>1$ afrecho, y a esto llama $<\mathrm{n}>$ acemite, y toma $<\mathrm{n}>\mathrm{aq}<\mathrm{u}>\mathrm{el}$ acemite y muelenlo ${ }^{20}$ muy bie $<\mathrm{n}>$ y esto es llamado adargama, y el pa $<\mathrm{n}>$ della es de muy gra $<\mathrm{n}>$ nudrimiento y de gran sustancia; pero porq $<\mathrm{u}>\mathrm{e}$ no $<\mathrm{n}>$ ay en ella afrecho ninguno es liuiano de moler y malo de salir $(27 \mathrm{v}-28 \mathrm{r})$.

En este trozo, como en algún otro de los que se citen, lo importante ya no son solamente los nombres de las cosas, sino cómo se obtienen mediante el proceso de elaboración transmitido a través de generaciones.

Se llamaba "harina seca" la revuelta con "afrecho" (Nebrija [1495] 1989) obtenida del trigo seco molido. El "almodón", arabismo no inventariado por Maíllo Salgado (1998), y cuya primera doc., según el DCECH, data de 1527, en las Ordenanzas de Sevilla, lo que está muy lejos de ser cierto (SMed. 1384), se hace remojando el trigo y moliéndolo ligeramente; la harina así obtenida se limpia de las granzas grandes, quedándose sólo con el "afrecho" 'cascarilla' o 'corteza' del grano, por otro nombre "salvado" (Nebrija [1495] 1989). Se le daba el nombre de "adárgama", otro arabismo no recogido por Maíllo, cuya primera doc. se remonta, también según el DCECH a 1253, pero sin indicar la referencia documental correspondien$\mathrm{te}^{21}$, a la harina que se sacaba del trigo remojado y gruesamente molido ("almodón") y que se limpiaba del "afrecho" y las "granzas"; a esto se le daba el nombre de "acemite" (Nebrija [1495] 1989), cuya, a su vez, prolongada molienda da una harina finísima, que es la "adárgama" o "flor de la harina", conocida a partir de finales del xv con el italianismo sémola, recogido por Palencia ([1490] 1967: 84b 'semula' s.v. colliride) y por Nebrija [1492] 1979 s.v. simila 'por el acemite o semola' y Nebrija [1495] 1989 s.v. acemite 'simila').

\footnotetext{
${ }^{13}$ remojado: remojada

${ }^{14}$ molido: molida

15 fincan: ffnca $<n>$

${ }^{16}$ aq<ue>llos: aq<ue>llas

${ }^{17}$ gra<n>os: gra $<$ n $>$ ças

18 limpios: limpias

19 descortezados: descortezadas

${ }^{20}$ muelenlo: muelelo

${ }^{21}$ Se trata, sin ningún género de duda, del Libro de los engaños e los asayamientos de las mugeres: "nos dixo que tomasemos farina de adargama e que la amasasemos con manteca" (Vuolo 1980: 21); "que tenie dos panes de adargama" (Vuolo 1980: 21).
} 
El arabismo "acemite" ${ }^{22}$ aparece, pues, en nuestra lengua antes del Xv, en la Biblia romanceada, según la información ofrecida por el DCECH ${ }^{23}$. En resumen, para J. de Aviñón el "acemite" es, por lo tanto, harina sin nada de salvado; de la molienda del "acemite" se obtiene la "adárgama", harina finísima, lo que verdaderamente sería la "sémola". Al parecer, en tiempos de Nebrija, habiendo desaparecido de la circulación "adárgama" (término que no recoge, así como tampoco Covarrubias y Del Rosal), "acemite" se confunde con la "sémola"; los dos nombres, en consecuencia, designan, ahora, la misma clase de harina.

En adelante, en el uso se instaura la confusión: para Covarrubias, acemite es 'la flor de la harina'; para la Academia (Daut.[1726] 1976 s.v. azemite) es 'el salvado, u afrecho menudo, que tiene alguna parte corta de harina'.

En el DRAE, en la entrada acemite, se recogen cuatro acepciones; la cuarta dice así:

Granzas limpias y descortezadas del salvado, que quedan del grano remojado y molido gruesamente.

Esta definición está inspirada, y copiada, en parte, de la SMed., razón por lo que resulta ininteligible, ya que el texto de la misma presenta un notabilísimo grado de deturpación sintáctica; no obstante, darles sentido a las dos líneas leídas es un juego de niños:

Grano limpio de granzas, remojado y molido gruesamente, y descortezado del salvado.

Este es el texto de la edición de 1545:

el adargama es, remojado y molido gruessamente cada grano en tres partes o $\mathrm{q}<\mathrm{ua}>$ tro, y despues tiran dende aq $<\mathrm{u}>\mathrm{el}$ afrecho largo y gruesso, y finca $<\mathrm{n}>$ $\mathrm{aq}<\mathrm{u}>$ ellos gra $<\mathrm{n}>$ os limpios [de] gra $<\mathrm{n}>$ ças [f. 28r] y descortezados $\mathrm{d}<\mathrm{e}>$ l afrecho, y a esto llama $<\mathrm{n}>$ acemite;

versión castellana restaurada:

el adargama es, remojado y molido gruessamente cada grano en tres partes o $\mathrm{q}<\mathrm{ua}>$ tro, y despues tiran dende aq $<\mathrm{u}>\mathrm{el}$ afrecho largo y gruesso, y finca $<\mathrm{n}>$

${ }^{22}$ Tampoco recogido por Maíllo ( $\left.{ }^{3} 1998\right)$.

${ }^{23}$ Curiosamente, el DCECH cita a Juan de Aviñón s.v. afrecho: DCECH, $1^{\mathrm{a}}$ doc. LBA (h. 1330-50); pero no lo hace s.v. almodón, adárgama y acemite; pero es más curioso todavía que Juan de Aviñón y su obra no se alfabeticen en el índice bibliográfico de las fuentes utilizadas. Es posible que se trate de descuidos, pero más me inclino a pensar que los autores no han utilizado directamente la Sevillana medicina. Si lo hubieran hecho no habrían dejado de señalar que la primera doc. de almodón y acemite es la de esta obra, 1384. 
aq $<\mathrm{u}>$ ellos gra $<\mathrm{n}>\mathrm{O}$ limpios [de] granças [f. 28r] y descortezados $\mathrm{d}<\mathrm{e}>1$ afrecho, y a esto llama $<\mathrm{n}>$ acemite;

versión española:

la adárgama se obtiene remojando y quebrantando poco el trigo, cada grano en tres o cuatro partes, después se les quita el afrecho largo y grueso y los trocitos quedan limpios de las granzas [f. 28r] y pelados del afrecho, a lo que se le da el nombre de acemite.

En este trozo de la SMed., como en tantísimos otros de la misma, todo anda revuelto; en la realidad, el trigo, después de aventado, se criba, y con ello desaparecen las "granzas" que pudieran quedar; después de molido el trigo, se limpia la harina de la cascarilla, cascabillo, "afrecho" o "Salvado" con un cedazo de malla más espesa.

En el texto, se utiliza, pues, "afrecho" como sinónimo de "granza" 'residuos de paja larga y gruesa, espiga, grano sin descascarillar, etc., que quedan del trigo y la cebada cuando se avientan y criban' (DRAE s.v. gran$z a^{2}$ ), y de "Cascabillo" 'cáscara del grano de los cereales' (DRAE, s.v.).

La primera acepción de "acemite" del DRAE: 'Afrecho con alguna corta porción de harina', según el DAut. s.v. azemite, es la del siglo XviII: "pero el uso moderno de esta palabra no se toma en este significado, sino en el que queda expresado". Entiéndese moderno frente al que recoge Covarrubias: 'es la flor de la harina'. El cambio semántico de 'harina de flor' o 'flor de la harina' al de 'afrecho con poca harina', es llamativo, pues este último significado es el opuesto del de la raíz árabe ${ }^{24}$. Este cambio de significado podría deberse, más que a un desplazamiento extremo de significado, casi antitético, de la misma naturaleza que el que sufrió el latín algidum 'frío' en romance 'caliente', a un mal entendimiento del texto de las Ordenanzas de Sevilla $\left(1527\right.$ y 1632) ${ }^{25}$ en el que del "azemite" se trata:

y este trigo deue ser ahechado, y limpio, y mojado de aquella manera que entienden que deue ser para fazer adargama: y primeramente, se deue moler, y fazer todo azemite con cedaço abierto, y sacar de[l] la foja del trigo, y cernerse otra vez el azemite con otro cedaço mas espesso, y sacar del azemite la farina, que es molida, que llaman adutaque, y poner esta farina a parte, $y$ el azemite que fincare, deue ser limpio, y claro, en manera que no finque en el boja, ni afre-

${ }^{24}$ Más datos e información en Carrasco (1992).

25 Ordenanças de Sevilla. Qve por sv original, son aora nueuamente impressas, con licencia del señor Assistente, Por Andres Grande, Impressor de libros, Año de mil y seyscientos, y treynta y dos[...]. Edición e introducción del facsímil de Víctor Escolano y Fernando Villanueva Sandino (Sevilla, 1975). 
cho ninguno, y tornarse a moler otra vez y fazer del azemite harina bien molida: y deue ser cernida esta farina con el padron de la adargama (Ord. Sev. 1975: 74).

Respecto del arabismo "adutaque ${ }^{26}$, hay que precisar que ni es "harina de flor' ni su primera doc. data de 1726 ni su documentación es única (DCECH s.v.). Del texto se colige, que "adutaque" no es sinónimo de "adárgama" 'harina de flor', como ya hizo notar Eguilaz ([1886] 1974) s.v., por el momento en que se obtiene, respecto de "adárgama", y por el procedimiento ${ }^{27}$.

EL PAN

El catorzeno catamiento es en la manera de amassar ${ }^{28}$, ca della ay que se echa en ella mucha agua y fazese pan esponjoso y mollete y este ${ }^{29}$ tal es de gran gouierno, liuiano de moler y deciende ayna del estomago; y el pan souado [con farina] es el contrario, que es malo de moler y sale tarde del estomago y cierra los caños, pero es de gran nudrimie $<\mathrm{n}>$ to, y pertenesce mas, tal pan como este, a los que afanan cada dia, que non para los folgados (28r).

Se ha visto, en el texto primeramente citado, que el alimento y facilidad de digestión del pan depende de la naturaleza de la harina con se haga: el hecho con "harina seca", por estar revuelta con "afrecho", se digiere y se elimina con facilidad, pero no es de tanto alimento como el hecho con las otras clases de harina; el pan de "almodón" es sabroso y muy bueno, y, por llevar "afrecho" también, es fácil de digerir y es el que comúnmente se consume; el de "adárgama" es de gran alimento, pero, aunque no tiene "afrecho", es fácil de digerir, pero difícil de eliminar.

De la manera de amasar la harina, depende la consistencia del pan; así, pues, lo hay "esponjoso y mollete", si se amasa con mucha agua; lo hay "Sobado", el que se hace añadiéndole a la masa aceite o manteca ${ }^{30}$, que es de digestión lenta, tarda en salir del estómago y cierra los conductos; es el que consumen los que tienen trabajos duros.

Al principio, desde la más antigua doc. que tengo, ésta de la SMed., 1384 (DCECH s.v. muelle, $1 .^{\underline{a}}$ doc. Nebrija [1495] 1989), se hablaba de "pan

${ }^{26}$ Tampoco recogido por Maíllo ( $\left.{ }^{3} 1998\right)$.

${ }^{27}$ El texto aducido por Eguílaz, aunque reproduce parcialmente el mismo que yo de la Ordenanza sevillana, tiene todas las trazas de estar modernizado, no por él, claro está, pero, a pesar de ello, se llega a la misma conclusión.

28 amassar: amasar

29 este: esto

30 Libro de los engaños e los asayamientos de las mugeres (1980: 21): "nos dixo que tomasemos farina de adargama e que la amasasemos con mantecan. 
mollete" ${ }^{31}$ y de "pan sobado" ${ }^{32}$; en el curso del tiempo, lo que era adjetivo pasó, como frecuentemente ocurre, a ser sustantivo común, "mollete" y "sobado". En el Glosario del Escorial (h. 1400) s.v. libum, -i, "por bodigo o pan mollete" (Castro 1936); A. Chirino, Menor daño (1 ${ }^{\text {er }}$ tercio s. XV): "e carnes tiernas e pan mollete e vaço e masado con anis e finojo" (Quirino 1973: 170, lín. 19); Sebastián de Horozco (entre 1558-1580): “a de yr al fin a parar / al arca de los molletes" (Horozco 1986); Francisco Hernández, trad. de la Naturalis Historia (1576: II, 320a): “Otras diferencias se toman de su forma y ansi hay tortas, panes grandes, medianos y pequeños, roscas, bollos, sovados y molletes; Juan de Salinas, Poesias (h. 1600): “les daba en sus banquetes / mas blancos que la leche los molletes" (1988: 269, v. 244); Bernardo J. de Alderete (1614: 1. II, ii, 229): "LACHMA. Atheneo: Apud Graecos panis quidam mollis, vocatur, conditurque lacte pauco, oleo \& sale quantum satis est. [...]. Este pan es el que llamamos mollete; por último, para abreviar la relación, un texto de Francisco Rodríguez Marín ([1882] 21981: I, 73,): "Cayó un moyete / Me dio en los dientes: / Mejor pa mí, / Que me lo comín.

El "mollete", al igual que el "Sobado", se hacían con "harina de flor"; el "sobado" siempre es redondo; el "mollete" puede ser ovalado o redondo; en la actualidad, siempre es redondo, al menos, en la Andalucía que yo conozco. El "mollete" es un panecillo de escasa altura, muy blanco y tierno, que se toma, especialmente, en el desayuno.

Respecto de su origen, pienso que no tiene por qué proceder necesariamente del fr. mollet (DCECH s.v. muelle): 1.ㅇ) porque puede tratarse de un andalucismo (toda la doc. que poseo, por ahora, es de andaluces, salvo la de Francisco Hernández, toledano, pero que, durante años, ejerció la medicina en Sevilla); 2.9 ) porque nada impide que "mollete" descienda de "molla", e incluso de "muelle", toda vez que cuando el acento que produjo la diptongación se desplaza a otra sílaba por razones morfológicas de flexión (quiero / queremos), o de derivación (tierno, terneza, ternura), y 3.9) porque el sufijo diminutivo -ete es productivo en español: serete, cachete, pinete, ajete, regordete, sorbete, ojete, etc. ${ }^{33}$; respecto de que en Nebrija (1495) se encuentre la primera datación, es evidente que no.

31 «Pan mollete" lo registra la Academia en 1734 (DAut. s.v. pan); en el DRAE, "mollete" no tiene entrada como sustantivo hasta ${ }^{19} 1970$. Nebrija (1495), sustantivo: mollete pan muelle. panis mollis.

32 "Pan sobado" lo registra la Academia en 1737 (DAut. s.v. pan); en el DRAE, "SObado", como sustantivo, no tiene entrada hata ${ }^{22} 2001$. Núñez de Taboada (1825), s.v. pan. Terreros (1788) s.v. sobados 'especie de tortas que usan en La Mancha'.

${ }^{33}$ Respecto del sufijo - ete en la lengua medieval castellana, vid. González Ollé 1972: 309-312. 
el gran pan tiene mucho meollo y la corteza delgada (28r);

$\mathrm{y}$ el pan pequeño es delgado y tiene poco meollo $\mathrm{y}$, por tanto, es de poco nudrimiento y flaco y sale tarde del estomago y estriñe el vientre; y el comunal es muy bueno; y este tal sera, a la comunaleza, de quatro onças y que sea mollete, y [f. 28v] de vn dia en otro, ca este es mejor $\mathrm{q}<\mathrm{u}>\mathrm{e}$ non el de las gra $<\mathrm{n}>$ des fogaças $\mathrm{q}<\mathrm{u}>\mathrm{e}$ viene $<\mathrm{n}>$ de Alcala de Guadayra y de Sant Lucar (28v).

Lo único interesante en este texto es el empleo de "meollo" con significado de 'miga'. Cosa anecdótica es saber que Alcalá de Guadaira era el pueblo que, hasta la guerra civil, muy probablemente, siempre ha abastecido de pan a Sevilla; era, y probablemente sigue siendo, un pueblo de tahonas y panaderos.

El diez y siete catamie $<\mathrm{n}>$ to es ser muy leudo o poco, ca el muy leudo es mas liuiano de moler en el cuerpo y deciende mas ayna del estomago, pero non es de $\tan$ gra $<\mathrm{n}>$ gouierno como el $\mathrm{q}<\mathrm{u}>\mathrm{es}$ oliuado, ca este es $\mathrm{d}<\mathrm{e}>$ mayor gouierno y sale mas tarde del estomago y atapa y engendra humores gruessos y viscosos, y el $\mathrm{q}<\mathrm{u}>\mathrm{e}$ es leudo, en comunal ${ }^{34}$, esse ${ }^{35}$ es el bueno (28v).

El pan muy subido (leudo 'levantado' <LEVITUM <LEVATUM), porque el exceso de levadura en la masa la ha hecho fermentar demasiado, es fácilmente digerible, pero no es de tanto alimento como el "olivado", el hecho con una masa que, por haberse enfriado, al ser metida en el horno, hace burbujas ${ }^{36}$.

El pan olivado no gozaba de estimación en Sevilla:

y el pan que fallaren menguado, o mal cocho, o oliuado, aqui en la cibdad, que sea para los presos de la carcel, y en los lugares de los terminos, que sea para los Almotacenes (Ord. Sev.[1632]1975,74v).

Hay otras clases de pan: pan cenceño 'ázimo' y viscocho (92r); pan de farina de ceuada (38v); pan de mijo, o su semejante (95r).

DEL VINO

No voy a tratar aquí del vino en el sentido usual del término, sino de los vinos medicamentosos, es decir, de los que se toman con finalidad curativa. De los otros vinos, según el parecer de Aviñón, trataré en otro momento.

\section{${ }^{34}$ comunal: comunales}

35 esse: esso

${ }^{36}$ DAut., s.v. olivarse (1737); olivarse ya no está en ${ }^{20} 1984$; la acepción que aquí interesa se encuentra s.v. olivar ${ }^{2}$, 2. Alcalá, 1505. 


\title{
El otro alfajor
}

Es sabido de todos que entre los dulces de la Pascua de Navidad, en Andalucía, está el "alfajor", llamado en otras zonas peninsulares de lengua española "alajú", sin que esto quiera decir que este último nombre no fuera utilizado aquí, como lo demuestran los testimonios del sevillano Mateo Alemán (Alvar 1992) y del malagueño Serafín Estébanez Calderón:

\begin{abstract}
Al lado de los dulces laboriosamente confeccionados y sobrecargados de esencias y perfumes, regalo solo del rico, se encuentra el acitrón, el alajú, los turrones y otros mil azúcares todavía de raza mora, que por módico precio procuran igual sabrosa satisfacción a la aldeana, al rústico y demás gente menuda ([1847] 1983: 67).

La alcorza, el alajú y el alfajó entre pañizuelos blancos y en canastillos muy lindos ([1847] 1983: 269).
\end{abstract}

También dan noticia de su existencia en Andalucía, entre otros, el cordobés del Rosal y García Lorca:

Para conocer la Alhambra de Granada, por ejemplo, antes de recorrer sus patios y sus salas, es mucho más útil, más pedagógico, comer el delicioso alfajor de Zafra o las tortas alajú de las monjas de Santiago (1997: 114) ${ }^{37}$.

Tanto el uno como el otro nombre designan tanto la masa como el dulce que de ella se hace; no obstante, y a juzgar por algunos textos, parece que, preferentemente, "alfajor" designaba el dulce, y "alajú" la pasta o masa de que este se hacía, o una torta, de cuyos ingredientes da noticia el DRAE s.v. No es de este dulce navideño tan conocido, sin embargo, del que aquí voy a ocuparme brevemente. En relación con su etimología, me parece fundada la concesión hecha por Maílo ( ${ }^{3} 1998$ : s.v. 92): "en el supuesto que ambos términos viniesen del mismo étimon". Por cuanto a la $-r$ final se refiere, la suposición de Corriente de que "alfajor" proceda de fa ur 'néctar' del persa af or 'jugo' (Maíllo, 92) parece viable, fonética y semánticamente para "alfajor" 'vino o bebida medicinal', pero menos, semánticamente, para ambos sinónimos 'dulce' ("alfajor") y 'masa de que se hace el dulce'("alajún). Tengo una curiosa papeleta en la que se lee "alajud" 'dulce morisco' (Villalba [1577] 1886: 23, 280).

Con este nombre de "alfaxor" (DAut 1726: s.v.; Minsheu ([1599] 2000), se designaba también una especial clase de vino o, con más precisión,

${ }^{37}$ En la edición de Obras Completas. de Aguilar, el texto está mutilado, no por la censura, porque lo que falta es absolutamente inocuo social y políticamente, sino por la imprenta: "el delicioso alfajor de Zafra o las tortas alaju de las monjas" (p. 49). 
de una bebida medicinal cuyo principal componente era el mosto. Al parecer de Aviñón, se trataba más de una medicina que de un alimento.

Esta es la receta de confección:

El noueno catamie $<$ n $>$ to es segu $<$ n $>$ el co $<$ m $>$ ponimiento del vino, ca ay de muchas maneras y son estas que se siguen:

El alfaxor es calle $<\mathrm{n}>\mathrm{te}$ y seco en tercero grado y es mas medecinal $\mathrm{q}<\mathrm{u}>\mathrm{e}$ no $<\mathrm{n}>$ nutritiuo ${ }^{38}$; y abre los caños, y esfuerça y ayuda a moler la via $<\mathrm{n}>\mathrm{da}$, siendo beuido por melezina, mas no $<\mathrm{n}>$ por vino; y esta es la recepta $\mathrm{d}<\mathrm{e}>\mathrm{l}$ alfaxor.

Mosto puro, vna arroua; fojas ${ }^{39} \mathrm{~d}<\mathrm{e}>$ [f. 64v] albahaca, $\mathrm{q}<\mathrm{ua}>$ tro onças; fojas de $m a<n>$ çanas, vna libra; me $<$ m $>$ brillos buenos y de bue $<$ n $>$ olor, mo $<$ n $>$ dados $\mathrm{d}<\mathrm{e}>\mathrm{p}<$ ar $>$ te de de $<\mathrm{n}>$ tro y partidos ${ }^{40}$ cada vno en ocho pedaços, dos libras $\mathrm{d}<$ e $>$ llos; y sea cozido en ca $<$ n $>$ dela ma $<$ n $>$ sa y clara fasta $\mathrm{q}<\mathrm{u}>\mathrm{e}$ me $<$ n $>$ gue la tercia $\mathrm{p}<$ ar $>$ te, medido por vna caña señalada en tres $\mathrm{p}<$ ar $>$ tes $\mathrm{d}<\mathrm{e}>\mathrm{l}$ altura de la caldera $\mathrm{y}, \mathrm{d}<\mathrm{e}>$ spues, tire $<\mathrm{n}>\mathrm{lo}^{41} \mathrm{~d}<\mathrm{e}>1$ fuego; y espume $<\mathrm{n}>\mathrm{lo}^{42}$ y dexe $<\mathrm{n}>\mathrm{lo}^{43}$ resfriar y ponganlo ${ }^{44}$ en vna tinaja en $\mathrm{q}<\mathrm{u}>\mathrm{e} \mathrm{q}<\mathrm{u}>$ epan diez arrouas; $\mathrm{y}$, a este respecto, faga $<\mathrm{n}>$ della $\mathrm{q}<\mathrm{u}>\mathrm{e} \mathrm{q}<\mathrm{u}>\operatorname{epan}^{45}$ doze arrouas, y dexen vn palmo vazio, porq $<\mathrm{u}>\mathrm{e}$ no $<\mathrm{n}>$ se salga en el feruor $\mathrm{q}<\mathrm{ua}>$ ndo firuiere; $\mathrm{y}$ echen cinco pesos de ma $<\mathrm{n}>$ çanas hechos quatro quartos, porq $<\mathrm{u}>\mathrm{e}$ tire $<\mathrm{n}>$ la orrura y por dar buen olor; y dexenlo ${ }^{46}$ tres dias y, despues, al quarto, espumenlo ${ }^{47}$ y alimpien aquella boca; y, despues, tome $<\mathrm{n}>$ canela fina, vna onça; y de gengibre, media onça; $y$ galangal y pimie $<n>$ ta luenga y redonda y clauos de girofre $y$ nuez moxcada $y$ nuez de xarca ${ }^{48}$ [?] y espiquenardi y macis y amoradux; de cada vno, q<ua>rta de onça; mosq $<\mathrm{u}>$ ete fino, vn adarme; $\mathrm{y}$ sea $<\mathrm{n}>$ molidas estas cosas $\mathrm{y}$, atadas en vn paño de lino, y colgadas ${ }^{49}$ en aq<u>ella tinaja, y sale marauilloso alfaxor $y$ de buen sabor; y el $\mathrm{q}<\mathrm{u}>\mathrm{e}$ quisiere $\mathrm{q}<\mathrm{u}>\mathrm{e}$ sea mas callente, puede acrecentar torongil y yerua de huerto y laurel y saluia y romero y niepta y sus semeja $<n>t e s ; y, ~ s i$ $\mathrm{q}<\mathrm{u}>$ isiere $\mathrm{q}<\mathrm{u}>\mathrm{e}$ sea laxatiuo, acrecie $<\mathrm{n}>\mathrm{te}$, en el cozer, sen y polipodio; $\mathrm{d}<\mathrm{e}>$ cada vno, q<ua>tro libras (64r).

No tendría nada de particular que el nombre "alfajor" del dulce se diera también al vino-medicina, a causa de la cantidad de ingredientes comu-

\footnotetext{
38 nutritiuo: nutratiuo

39 fojas: foja

${ }^{40}$ partidos: parados

${ }^{41}$ tire $<\mathrm{n}>$ lo: tire $<\mathrm{n}>\mathrm{la}$

${ }^{42}$ espume $<n>$ lo: espume $<n>l a$

${ }^{43}$ dexe $<$ n $>$ lo: dexe $<$ n $>$ la

${ }^{44}$ ponganlo: ponganla

${ }^{45}$ q<ue>pan: qu<ue>pa

${ }^{46}$ dexenlo: dexenlas

47 espumenlo: espumenla

48 xarca: xarta

${ }^{49}$ colgadas: colgado
} 
nes que entran en su elaboración, si bien la receta de Juan de Aviñón es bastante más abundosa de ellos.

La más antigua documentación de "alfajor" 'vino-medicina' conocida del DAut. s.v., y recogida, después, por el DCECH, s.v. data de 1564; la autoridad citada es el sevillano Alonso de Fuentes, autor de la traducción al español del Asno de oro:

El vino de alfajor, que es vino de los Dioses, ministraba Ganimedes a Júpiter.

La documentación que suministra la SMed. es 180 años anterior. El DHist. (1933) y el DHLE s.v. desconocen el texto de Aviñón; no obstante, este último diccionario da doc. anterior a la de 1384; se encuentra en la Visión de Filiberto c. 1330 (ZRPh 2 [1878]: 40-60). Es de hacer notar que el editor ha entendido, correctamente, que en el texto "alfajor" se refiere al dulce: 'cierta pasta que hacen los moros con pan rallado, miel, alegría y especias', frente al DHLE s.v. 1., que piensa que se refiere a la bebida medicinal hecha con "mosto puro":

sy te pedia vyno blanco dauas me tu alfaxor e buen vino bermejo e fartauas me muy bien (54 y n. 1);

lo que en el texto se opone a "vino blanco es "vino bermejo", a lo que añadía el dulce; es decir, le daba vino y comida, hasta hartarse.

La denominación "vino pimente" se le daba a un vino aromatizado con especias.

Respecto de que "pimente" sea préstamo del francés o que haya ocurrido lo contrario, todo depende de la fecha de su primera datación, pero en esto tampoco hay seguridad. Según Bloch-Wartburg, en fr. "piment" "vino aromatizado' con especias, como lo declara el texto de Aviñón, es un préstamo de una lengua meridional (cat., prov. o it.), cuya $1 . \underline{a}$ doc. sería piument de h. 1270 (Bloch y von Wartburg ${ }^{6} 1975$ : s.v. piment); en cast. med., según el DCECH s.v. pimienta, en nuestra lengua procedería del fr., cuya 1. ${ }^{\mathrm{a}}$ doc., a juicio de Gamillscheg ( ${ }^{2} 1969$ : s.v. piment), en la forma citada de piument, sería del siglo XII.

El .x. catamie $<\mathrm{n}>$ to es del vino pimente, ca el vino pime $<\mathrm{n}>\mathrm{te}$ es bueno $\mathrm{p}<\mathrm{ar}>\mathrm{a}$ los flematicos; $\mathrm{y} p<\mathrm{ar}>\mathrm{a}$ el inuierno, $\mathrm{q}<\mathrm{u}>\mathrm{e}$ es frio y beuido por fruta, ca es callente y seco en tercero grado; y abre los caños, y suelta la orina, y esfuerça las virtudes todas, beuie $<$ n $>$ dolo te $<$ m $>$ pladame $<$ n $>$ te; y esta es su recepta:

Recipe: ge $<$ n $>$ gibre, $\mathrm{o}<\mathrm{n}>$ ça y $\mathrm{q}<\mathrm{ua}>\mathrm{rta}$; pimie $<\mathrm{n}>\mathrm{ta}$, vna onça; pebre, luego, tres ochauas; garangal, nuez moxcada y flor [f. 65r] de canela y clauos y cubebas; de cada vno, q<ua $>$ rta de onça; nuez de especia, juncia auellanada, espiq<u $>$ enardi, febro [?], macis; $d<e>$ cada vno, vna ochaua; sea $<n>$ fechos poluos y, en vna onça $\mathrm{d}<\mathrm{e}>$ stos poluos, $\mathrm{po}<\mathrm{n}>$ ga $<$ n $>$.v. libras de vino bla $<$ n $>$ co o bermejo y vna libra 
$\mathrm{d}<\mathrm{e}>$ miel espumada; y buelua $<\mathrm{n}>$ lo todo, y fierua vn poco la miel $\operatorname{co}<\mathrm{n}>\operatorname{los}$ poluos y $\operatorname{co}<\mathrm{n}>$ vn cotrofe $\mathrm{d}<\mathrm{e}>$ vino; $\mathrm{y}, \mathrm{d}<\mathrm{e}>$ spues $\mathrm{q}<\mathrm{u}>\mathrm{e}$ fuere firuie $<\mathrm{n}>\mathrm{te}$, eche $<$ n $>$ aq $<$ u $>$ ello todo $c o<$ n $>$ el vino y mezcle $<$ n $>$ lo muy bien y, $\mathrm{q}<$ ua $>$ ndo fuere apurado, beua $<\mathrm{n}>\mathrm{lo}(64 \mathrm{v})$.

La "clarea", por ser una bebida aromática, se parece al "vino pimente", pero, entre uno y otra, hay una diferencia notable: en un caso, se hace con vino blanco; en otro, la "clarea", con vino tinto; los ingredientes no son muy distintos:

El catamie $<\mathrm{n}>$ to. $\mathrm{xj}$., $\mathrm{e}<\mathrm{n}>$ la clarea. La clarea es cerca $\mathrm{d}<\mathrm{e}>\mathrm{l}$ vino pimente, mas no $<$ n $>$ es $\operatorname{ta}<$ n $>$ calle $<$ n $>$ te; y esta es su recepta:

Recipe: canela y gengibre; de cada vno, media libra; gaudingal, pimienta y clauos y nuez de xarca ${ }^{50}$ [?]; d<e $>$ cada vno, vna onça; flor de canela, dos adarmes; nuez moxcada y amoradux y cardamomo; $\mathrm{d}<\mathrm{e}>$ cada vno, media $\mathrm{o}<\mathrm{n}>$ ça; espiq $<\mathrm{u}>\mathrm{e}$, vn adarme. Sean fechos poluos y, en vna onça destos poluos, po $<$ n $>$ ga $<$ n $>$ libra y media de açucar bla $<\mathrm{n}>$ co y siete de bue $<\mathrm{n}>$ vino bermejo, y mezcle $<\mathrm{n}>$ lo todo, y cuele $<$ n $>$ lo $c o<n>$ vn poco de estameña fasta $\mathrm{q}<\mathrm{u}>\mathrm{e}$ sea claro $(65 \mathrm{r}$ ).

En el DCECH s.v. claro, se estima que el nombre de esta bebida olorosa puede estar tomado del fr. claré; están a favor de este parecer dos cosas: 1) la 1. a doc. en fr. es del siglo xii (Greimas 1968: s.v.); 2) resulta imposible considerar que de claro pueda derivar "clarea"; en tanto que es casi evidente que la vocal final $a$ es el modo expedito y acertado de castellanizar el galicismo.

Sorprendentemente, en esta receta médica no se indica la clase de enfermos a que beneficiaría.

Quiero creer que la clase de vino medicamentoso llamado "vino florido", recibe el nombre de uno de los ingredientes que en su confección figuran: la "flor del saúco", la "flor del cohombro", la "flor del cantueso", y la "flor de las borrajas". No tengo doc. anterior o posterior a esta de Aviñón:

El catamiento dozeno, en el vino florido; este vino es bueno $\mathrm{p}<\mathrm{ar}>\mathrm{a}$ los flematicos y $\mathrm{p}<\mathrm{ar}>\mathrm{a}$ los restreñidos y $\mathrm{p}<\mathrm{ar}>\mathrm{a}$ los ydropicos, y abre las opilacio $<\mathrm{n}>\mathrm{es}$, y purga la flema de las coyunturas; esta es su recepta:

Flor de sahuco, vna libra; flor de cogombros amargos, media libra; flor de cantueso, q<ua>rta de libra; flor de borrajas, ochaua de libra; turbite, vna libra; sen y polipodio; de cada vno, vna libra; passas ${ }^{51}$, matalahuua, finojo; de cada vno, dos $\mathrm{o}<\mathrm{n}>$ ças; asaro ${ }^{52}$, espiq $<\mathrm{u}>\mathrm{e}$ y carlina ${ }^{53}$ y esq $<\mathrm{u}>$ ena $<\mathrm{n}>$ te y clauos; de cada vno, vna $\mathrm{o}<\mathrm{n}>$ ça; miel rosada, dos libras; açucar, dos libras; vino bla $<\mathrm{n}>\mathrm{co}, \mathrm{q}<\mathrm{ua}>$ tro

\footnotetext{
50 xarca: yxarta

51 passas: pasas

52 asaro: asera

53 carlina: casalina
} 
açu $<$ m $>$ bres. Sea $<\mathrm{n}>$ todas estas cosas machucadas y remojadas en vno y, $\mathrm{d}<\mathrm{e}>$ spues, colado, ta $<\mathrm{n}>$ to fasta $\mathrm{q}<\mathrm{u}>\mathrm{e}$ sea claro, y tome $<\mathrm{n}>\mathrm{d}<\mathrm{e}>$ llo vn cotrofe por la mañana [f. $65 \mathrm{v}$ ] y dara aq $<\mathrm{u}>\mathrm{el}$ dia dos camaras $(65 \mathrm{r}-65 \mathrm{v})$.

"Vino cocido", "mosto cocido" y "arrope" (Pemartín 1965: s.v.) son sinónimos con los que se denomina esta especie de jarabe. El secreto de su elaboración está en que las uvas han de ser muy dulces:

El .xiij. catamie $<\mathrm{n}>$ to en $<\mathrm{e}>1$ vino cozido, que dize $<\mathrm{n}>$ arrope, este ${ }^{54}$ es calle $<\mathrm{n}>\mathrm{te}$ en tercero grado y seco en $<\mathrm{e}>1$ segu $<\mathrm{n}>\mathrm{do}$; y cierra los caños $\mathrm{d}<\mathrm{e}>1$ figado y abre los caños de los pechos y de los bofes; y ay del co $<m>$ puesto; es muy virtuoso $\mathrm{p}<\mathrm{ar}>\mathrm{a}$ la tosse y $\mathrm{p}<\mathrm{ar}>\mathrm{a}$ la seq $<\mathrm{ue}>\mathrm{dad}$, y es abridero $\mathrm{d}<\mathrm{e}>1$ figado $\mathrm{y} \mathrm{d}<\mathrm{e}>1$ baço; y esta es su recepta:

Arrope de vuas dulces y limpias del boruio, a mano, y non esprimie $<\mathrm{n}>\mathrm{do}$, vna arroua; ysopo, marrubio ve $<\mathrm{n}>$ toso, cula $<\mathrm{n}>$ trillo $\mathrm{d}<\mathrm{e}>1$ pozo, rayz de finojo y de cardo corredor y lirio cardeno; de cada vno, media libra; passas ${ }^{55}$ mo $<n>$ dadas, dos libras; oroçuz y alq<ue>tira; de cada vno, vna onça; cubebas, vna onça; sea fecho arrope $(65 \mathrm{v})$.

Esta de la SMed. es la primera doc., por ahora, no la del Glos. del Esc., h. 1400 (DCECH, s.v.). No habría por qué recordar que "arrope" es un arabismo ${ }^{56}$.

Nada especial hay que decir del nombre "vino laxativo" y, mucho menos, de la finalidad de su confección:

Recepta de vino laxatiuo: tomen la recepta del vino pimente o de la clarea y buelua $<\mathrm{n}>$ letuario de çumo $\mathrm{d}<\mathrm{e}>$ rosas y diasen o diamagna y cuelenlo, y dara camaras, segun la contia que echaren $(80 \mathrm{v})$.

\section{BIBLIOGRAFÍA CITADA}

AlCAlÁ Venceslada, A. 1980. Vocabulario andaluz. Madrid: Gredos.

ALDETERE, B. J. de. 1614. Varias antiguedades de España y Africa y otras provincias. Amberes: Iuan Hafrey.

AlONSO DE HeRRERA, G. 1981. Agricultura general [...]. Edición crítica de Eloy Terrón. Madrid: Servicio de Publicaciones, Ministerio de Agricultura.

ALVAR, M. 1992. "Alaju( $r$ ) y alfajor en Mateo Alemán y su reflejo en la geografía lingüistica actual,, en Estudios Léxicos. Segunda serie: 71-81. Madison: Seminary of Medieval Studies.

AviNón, J. de. 1545. Seuillana medici/na. Que trata el modo connseruati/uo y curatiuo de los q<ue $>$ abita<n> en la/ muy insigne ciudad de Seuillal la $q<u a>l$ sirue $y$

\footnotetext{
54 este: esto

55 passas: pasas.

56 Tampoco recogido en Maíllo ( $\left.{ }^{3} 1998\right)$.
} 
ap<ro>uecha $p<a r>a$ a $q$ ua $>$ lquier/ otro lugar destos reynos. Obra/ $a<n>$ tigua digna $d<e>$ ser leyda. Ua di/rigida al illustrissimo cabildo $d<e>>$ la misma ciudad. Año. MDXLV. Hay edición facsímil de Alfonso de Rembrandt. 1995. Alicante: Editions, S.A.

- 2000. Sevillana Medicina. Introducción, edición, versión y notas de José Mondéjar. Madrid: Arco Libros.

Bloch, O. y W. VON WARTBURG. ${ }^{6} 1975$. Dictionnaire étymologique de la langue française. París: Presses Universitaires de France.

CARrasco, P. 1992. "Los andalucismos léxicos en la obra del cordobés Francisco del Rosal, en M. Ariza et al. (eds.), Actas del II Congreso Internacional de Historia de la Lengua Española: I, 971-979. Madrid: Pabellón de España, S.A.

Castro, A. [1936] ${ }^{2}$ 1991. Glosarios latino-españoles de la Edad Media. Madrid: Anejo XXVII de la RFE. También en Madrid: CSIC, Biblioteca de Filología Hispánica.

Covarrubias, S. de 1943. Tesoro de la Lengua Castellana o Española, según la impresión de 1611, con las adiciones de Benito Remigio Noydens publicadas en la de 1674. Edición preparada por Martín de Riquer. Barcelona: S. A. Horta.

Columela, L. J. M. 1977. De re rustica. Cambridge, Massachusetts, Londres: Loeb Classical Library, Harvard-Heinemann.

- 1988. De los trabajos del campo. Edición a cargo de Antonio Holgado Redondo, Madrid: Ministerio de Agricultura, Pesca y Alimentación.

EgUILAZ Y YANGUAS, L. de. [1886] 1974. Glosario etimológico de las palabras españolas [...] de origen oriental. Madrid: Atlas.

ESTÉBANEZ CALDERÓN, S. [1847] 1983. Escenas andaluzas. Madrid: Ediciones Atlas.

GAMILLSCHEG, E. ${ }^{2} 1969$. Etymologisches Wörterbuch der französischen Sprache. Heidelberg: Carl Winter, Universitätsverlag.

GarCía LoRCA, F. 1997. Conferencia: "Canciones de cuna españolas: Añadas. Arrolo. Nana. Vou-veri-vou", en Miguel García-Posada (ed.), Obras Completas, III. Prosa. Barcelona: Galaxia Gutenberg/Círculo de Lectores.

GONZÁlez Ollé, F. 1972. Los sufijos diminutivos en castellano medieval. Madrid: Anejo LXXV de la RFE, CSIC.

Greimas, A. J. 1968. Dictionnaire de l'ancien français jusqu'au milieu du XIV siècle. París: Larousse.

HERNÁNDEZ, F. 1976. Obras completas, V. Historia natural de Cayo Plinio Segundo, II. Trasladada y anotada por el doctor Francisco Hernández. México: Universidad Nacional de México.

HerReRA, M.a T. (dir.). 1996. Diccionario español de textos médicos antiguos. Madrid: Arco Libros.

HoROzCO, S. de. 1986. Teatro universal de proverbios. Edición, prólogo, índices y glosario de José Luis Alonso Hernández. Universidad de Groningen-Universidad de Salamanca.

LACARRA, M. ${ }^{3}$ J. (ed.). ${ }^{2} 1995$. Sendébar. Madrid: Cátedra.

LASSO DE LA VEGA y CORTEZO, J. [1891] 1988. Biografía y estudio crítico de las obras del médico Nicolás Monardes. Sevilla: Padilla Libros.

Maíllo SAlgado, F. ${ }^{3} 1998$. Los arabismos del castellano en la Baja Edad media Salamanca.

Minsheu, J. [1599] 2000. A Dictionarie in Spanish and English. Málaga: Universidad de Málaga.

NebrijA, E. A. de [1492] 1979. Diccionario Latino-Español (Salamanca, 1492). Estudio preliminar por Germán Colón y Amadeu-J Soberanas. Barcelona: Puvill Editor. 
- [1495] 1989. Vocabulario español-latino. Madrid: Real Academia Española.

PALENCIA, A. de [1490] 1967. Universal Vocabulario en Latin y en Romance. Reproducción facsimilar de la edición de Sevilla, 1490. Madrid: Comisión Permanente de la Asociación de Academias de la Lengua Española.

Pemartín, J. 1965. Diccionario del vino de Jerez. Barcelona: Gustavo Gili.

PLINIO. 1971. Naturalis Historia, V, Books XVII-XIX. Cambridge, Massachusetts-London: Loeb Classical Library, Harvard-Heinemann.

QuiRINo, A. 1973. Menor daño de la medicina. Edición crítica y glosario de María Teresa Herrera. Salamanca: Universidad de Salamanca.

RAE. [1726] 1976. Diccionario de autoridades, A-C. Edición facsímil, Madrid: Gredos. RODRíGuez MARÍN, F.1988. La verdadera biografía de Nicolás Monardes. Sevilla: Padilla Libros (Madrid: RABM, 1925).

- [1882] ${ }^{2} 1981$. Cantos populares españoles. Madrid: Atlas.

Rosal, F. del. [1601] 1992. Diccionario etimológico. Edición facsimilar y estudio de Enrique Gómez Aguado. Madrid: CSIC.

Salinas, J. de. 1988. Poesias humanas. Edición de Henry Bonneville. Madrid: Clásicos Castalia, 164.

SÁNChez MONGE y PARelladA, E. 1981. Diccionario de plantas agricolas, Madrid: Servicio de Publicaciones Agrarias, Ministerio de Agricultura.

Villalba y EsPaÑa, B. de. [1577] 1886. El pelegrino curioso y Grandezas de España. Ed. de Pascual de Gayangos. Madrid: Bibliófilos españoles. T. 23. Vol. I.

VuOlo, E. (ed.). 1980. Libro de los engaños e los asayamientos de las mugeres. Nápoles: Nuovo Medioevo, Liguori Editore. 